\title{
Literature review of Management and Quality of Life of Idiopathic Thrombocytopenic Purpura (ITP) patients
}

\author{
Article by Kavita Gupta \\ PhD Scholar at Texila American University, Guyana \\ E-mail:16kavitagupta@texilaconnect.com
}

\begin{abstract}
ITP scientifically known as 'Idiopathic Thrombocytopenic Purpura' is characterized as an uncommon benign acquired autoimmune hematologic disorder of unknown cause or etiology and normal bone marrow with acute condition in children and a chronic condition in adults which is often accompanied by symptoms such as, occasional Petechiae, Ecchymoses, excessive bleeding episodes in the form of Mucosal bleeding, spontaneous nosebleeds, bleeding from the gums, blood in the urine, blood in the stool, abnormally heavy menstruation, prolonged bleeding from cuts, and profused bleeding during surgery, with a platelet count of less than $100 \times 109 / L(100,000 / \mu \mathrm{L})$. Chronic ITP was observed to be a rare disorder found to be associated with a loss of tolerance to platelet antigens and a phenotype of accelerated platelet destruction and impaired platelet production. According to the recent studies, prevalence of 1 in 5.000 was estimated with prevalence in adults and children ranging from 9.6 to 189 per 100000 person ultimately leading ITP to be an orphan disease. Moreover, the increased risk of death was observed largely concentrated in patients 60 years of age or older. Mortality rate was observed to be very low with most of the outpatients being treated. ITP could present either alone (primary) or in the setting of other conditions (secondary) such as infections or altered immune states. This chapter of reviewed article highlighted the aspects of current understanding of definition of ITP, Pathophysiology of ITP, Diagnosis of ITP with considerable clues from genetic studies, treatment of Primary ITP with immunomodulatory mechanisms of action in ITP therapies (Evidence-based from clinical trials.gov) along with the Quality-of-Life of the ITP patients.
\end{abstract}

Keywords: 'Immune Thrombocytopenic Purpura (ITP)'; 'Mortality'; 'Hemorrhage'; 'Thrombocytopenia,' 'Platelet Count,' 'Autoimmune Thrombocytopenic Purpura, ' Complete Blood Count,' 'Bone Marrow Examination,' 'Reticulocyte Count,' 'Antinuclear Antibody Test,' 'Pathophysiology,' 'Treatment,' 'Refractory disease,' 'Platelet response,' 'Thrombosis'.

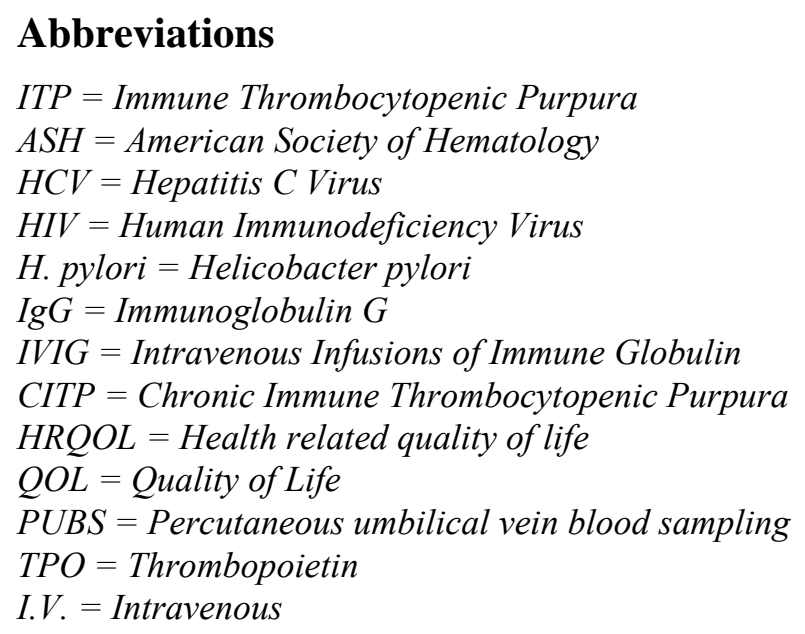


South American Journal of Clinical Research

Volume 3, Issue 1, 2016

\section{Introduction}

According to the practice guidelines introduced in 1996 by the American Society of Hematology (ASH) for the diagnosis and management of ITP stated 'Idiopathic Thrombocytopenic Purpura' as a benign acquired autoimmune hematologic disorder which was identified and evaluated by the physical examination, complete blood count, blood smear, reticulocyte count, Rh typing, direct antiglobulin (Coombs) test, and examination of the peripheral smear [1, 2]. ITP could be classified based on patient age (adult or childhood ITP), and duration of thrombocytopenia: newly diagnosed, up to 3 months from diagnosis; persistent, 3-12 months from the time of diagnosis; chronic, $>12$ months from the time of diagnosis[3]. The estimated annual incidence of adult ITP ranged from 0.6 to 6.6 cases per 100,000 adults [3]. Women were estiamted to be affected disproportionately, with a female to male ratio of nearly two to one [3]. In adults, the most prevalent infections associated with thrombocytopenia were those from hepatitis $\mathrm{C}$ virus (HCV), human immunodeficiency virus (HIV), and Helicobacter pylori (H. pylori) [4]. Overall, approximately $40 \%$ of patients with ITP were found to have a reduced platelet turnover [4]. Both in vitro and clinical studies identified that spleen was the primary site of antibody production [3, 4]. Luiken et al. and Hirschman and Shulman demonstrated that the transmissible agent in the blood was immunoglobulin, primarily immunoglobulin G (IgG) [1]. McMillan et al. And Chang et alreported that IgG produced by cells (grown in vitro) from the spleens of patients with ITP bound to megakaryocytes, whereas IgG produced by cells from the spleens of healthy controls did not bind to megakaryocyteswhich further supported the view that autoantibodies in ITP suppressedthe megakaryocyte production and maturation and platelet release[4]. It was observed that ITP might be caused due to either immune-mediated increased destruction of platelets or decreased production of platelets that involved the complex abnotrmalities of both the B-cell and the T-cell compartments which resulted in an overall decrease in circulating platelets [4]. Thus, pathogenesis of ITP was found to be caused by antibodies against platelet glycoproteins, most commonly platelet glycoprotein IIb/IIIa, the platelet fibrinogen receptor [1]. Filion et al. showed that CD4+CD25+ regulatory T-cells abnormalities emerged from the investigation of immune regulation in ITP patients [4].

Though, ITP was not found to be a genetic disorder, yet there were some evidences that supported ITP with genetic linkage. In a study performed by, Ermann \& Fathman, 2001 observed the presence of autoimmune disease in one out of 2 monozygotic identical twins [2]. In a study performed by Harrington and Hollingsworth in 1951 observed a child with purpura born to a mother with chronic ITP which resolved 3 weeks later, although the mother still had ITP $[1,4]$. The existence of a humoral anti-platelet factor that had been passed from mother to child was advanced [1, 4]. To test this hypothesis, Harrington received $500 \mathrm{~mL}$ of blood from a patient with ITP. Within three hours, his platelet counts dropped below 10x109/L as he developed chills, fever, headache, confusion and petechiae [1, 4]. His platelet count remained extremely low for four days, finally returned back to normal levels by the fifth day [1, 4]. A 2006 review of the Pediatric and Adult Registry of Chronic ITP (PARC-ITP) found that 10 out of 445 (2.2\%) of pediatric patients reported a positive family history of ITP [5]. It might be that in the future, characterization of genes by studying familial ITP would led to important clues into the pathogenesis of ITP [5].

The various treatment of idiopathic thrombocytopenic purpura was mostly performed with regard to the current practice guidelines according to which the approved drugs for recommendation included corticosteroids (i.e., prednisone) and/or intravenous infusions of immune globulin (IVIG), corticosteroids (i.e., prednisone) and/or intravenous infusions of immune globulin (IVIG), vincristine, azathioprine (Imuran), Danazol, cyclophosphamide, and cyclosporine with variable effectiveness in treating ITP and potential harmful side effects namely, facial swelling, hypertension, insomnia, hyperglycemia, cataract, weight gainfollowed by splenectomy in severe cases of excessive bleeding episodes[3, 6, 7]. 182 patients who underwent splenectomy in infancy and childhood were followed for periods of 2 to 15 years [8]. Serious infections occurred in 11 patients (6\%) with death in 6 (3.3\%) [8]. In 
10 patients the infection was sepsis, and in all but one patient the infection occurred within 2 years of splenectomy [8].

In a 18 month follow-up study performed by Cooper, et al. 2002based on questionnaire survey on 28 ITP patients of repeated infusions of anti-D, a marked improvement in general health, physical and emotional health was observed [3]. In another study performed by Matzdorff and Arnold, 2007, on 91 ITP patients through questionnaire survey it was observed that $75 \%$ of the ITP patients who received corticosteroid treatment experienced weight gain, moon face, anxiety and depression, restlessness and insomnia [3]. In a study performed by Cohen, et al., it was observed that a 30-year old woman lose 20.4 years of her potential life expectancy [3]. In a study performed by Zhou, et al. on 236 ITP patients through SF-36 form questionnaire it was observed that $88.1 \%$ of ITP patients had a fear of bleeding that significantly reduced their quality of life [3]. In a study performed by Mathias, et al. on ITP patients based on findings from the published literature, existing questionnaires, expert clinical opinion from leading hematologists, and input from focus groups it was observed that patients suffered from feelings of social embarrassment due to visible signs of the disease (bruising) and fatigue that ultimately prevented them from participating in any physical activity [3]. Moreover, most of the ITP patients encountered less ability to concentrate on work during office hours thereby, had a great impact on the work life of ITP patients along with anxiety, depression and fear of disease that had an emotional effect on their personal relationships [3]. Besides, both male and female participants in the focussed groups reported decreased libido due to the symptoms of ITP and the side effects of treatment [3].

\section{Aim}

The main objective of this review study was:

a. To understand the overview of aetiology, pathophysiology, epidemiology with regard to Idiopathic Thrombocytopenic Purpura for the treatment purposes worldwide.

b. To identify the improved and effective management of high-risk cases of ITP.

c. To identify and analyze the impact of ITP and its treatment on patients' health-related quality of life (HRQOL).

d. To assess clinical safety and efficacy of interventional treatment regimens in the management of symptoms being produced by Idiopathic Thrombocytopenic Purpura.

e. To identify and understand the various randomized clinical trials being conducted with respect to evaluate interventional therapies currently used in ITP with a targeted focus on measuring the benefits of therapy in terms of the clinical outcomes of bleeding and mortality, as well as the adverse effects of treatment.

\section{Search methodology}

The core text that was focused throughout the article write up was 'Idiopathic Thrombocytopenic Purpura'. All types of Randomized controlled studies and open labelled studies were included. Moreover, children, Adolescents and Adults suffering from Idiopathic Thrombocytopenic Purpura were included in this review. The methodology being adopted for the relevant literature review, searching the databases like, PUBMED, Cochrane Library, MEDLINE, EMBASE, Clinical trials.gov, abstracts from the American Societies of Hematology and Clinical Oncology annual meetings, and bibliographies of relevant articles and reviews were searched in database until June 2016 by using keywords such as, "immune thrombocytopenic purpura (ITP)"; "mortality"; "hemorrhage”; "Thrombocytopenia”, "Platelet Count," "Autoimmune Thrombocytopenic Purpura," "Complete Blood Count," "Bone Marrow Examination," "Reticulocyte Count," “Antinuclear Antibody Test," "Pathophysiology," "Treatment," "Refractory disease” was utilized. The computerized search retrieved 50articles. Moreover, a greater number of google searches were done to gather reliable and valid information from websites that primarily focussed on safety and efficacy ofConventional experimental interventions to be used in patients who suffered from Idiopathic Thrombocytopenic Purpura. Published abstracts were reviewed to identify those 
South American Journal of Clinical Research

Volume 3, Issue 1, 2016

that focused on the effect of ITP or ITP treatments. The search was confined to studies being published in English language. Moreover, PRISMA methodology was adopted for inclusion of relevant studies. Most of the ITP literature reviewed in this report pertained to therapy for the management of the underlined disease along with its impact on the quality of life on the people suffering.

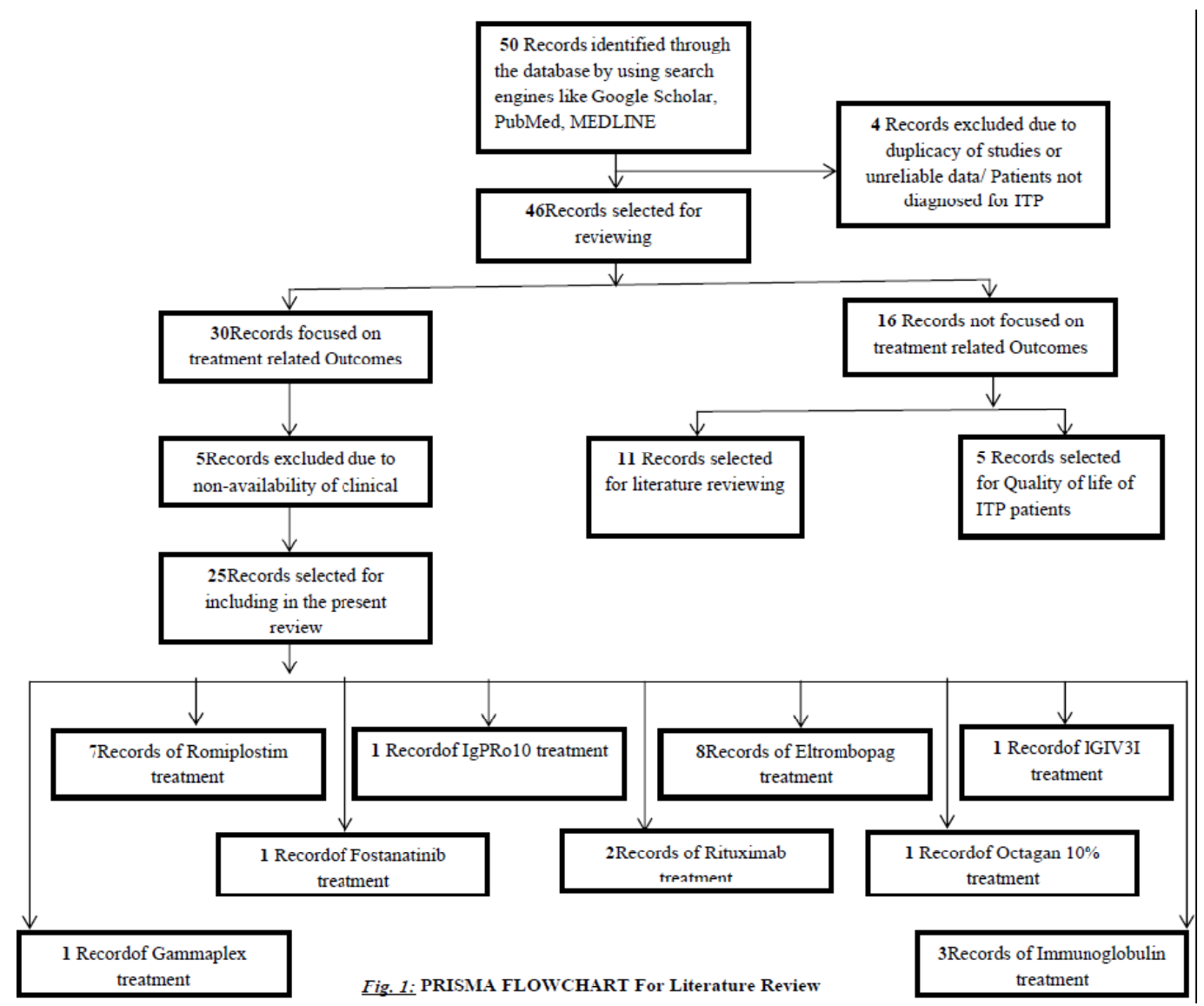

Fig. 1. PRISMA FLOWCHART For Literature Review

\section{Description of studies (clinical setting in humans)}


South American Journal of Clinical Research Volume 3, Issue 1, 2016

\begin{tabular}{|c|c|c|c|c|c|c|c|c|}
\hline S.N & Study period & Clinical Trial No. & Indication & Population & $\begin{array}{l}\text { Study } \\
\text { Design }\end{array}$ & $\begin{array}{l}\text { Intervention } \\
+ \\
\text { Route of } \\
\text { Administration }\end{array}$ & Results/Significance & Adverse Events \\
\hline 1. & $\begin{array}{l}\text { 2010-2016 } \\
\text { (24 months) }\end{array}$ & $\begin{array}{l}\text { NCT01143038 } \\
\text { [9] }\end{array}$ & ITP & $\begin{array}{l}\mathrm{N}=75 \\
\text { Adults } \\
\text { (Females:44 } \\
+ \\
\text { Males:31) }\end{array}$ & $\begin{array}{l}\text { Phase } 2 \\
\text { Open } \\
\text { label }\end{array}$ & $\begin{array}{l}\text { Romiplostim } \\
\text { subcutaneous } \\
\text { injection }\end{array}$ & $\begin{array}{l}\text { Well tolerated in } \\
\text { early stage ITP }\end{array}$ & $\begin{array}{l}\quad \text { (17S.A.E.+50A.E./75) } \\
\text { Thrombocytopenia, Atrial } \\
\text { Fibrillation, Hypothyroidism, } \\
\text { Abdominal Pain, Pleuritic Pain, } \\
\text { Tendon Rupture, Immune } \\
\text { Thrombocytopenic Purpura, Vertigo, } \\
\text { Conjunctivitis, Diarrhoea, fatigue, } \\
\text { headache, haematoma, Petechiae, } \\
\text { cough, influenza. }\end{array}$ \\
\hline 2. & $\begin{array}{l}\text { 2011-2015 } \\
\text { (Pharmacovigila } \\
\text { nce) }\end{array}$ & $\begin{array}{l}\text { NCT01390649 } \\
{[10]}\end{array}$ & ITP & $\begin{array}{l}\mathrm{N}=57 \\
\text { Adults } \\
\text { (Females:37 } \\
+ \\
\text { Males:20) }\end{array}$ & $\begin{array}{l}\text { Phase } 4 \\
\text { Open } \\
\text { label }\end{array}$ & $\begin{array}{l}\text { IgPro10 } \\
\text { Intravenously }\end{array}$ & $\begin{array}{l}\text { Set of Antibodies } \\
\text { frequently bound to } \\
\text { Red Blood Cells } \\
\text { (RBCs) in Subjects } \\
\text { Experiencing ITP }\end{array}$ & $\begin{array}{l}\text { (1 S.A.E +19 A.E /57) } \\
\text { Immune Thrombocytopenic Purpura, } \\
\text { Headache, Pyrexia. }\end{array}$ \\
\hline 3. & $\begin{array}{l}2010-2015 \\
\text { (2 years) }\end{array}$ & $\begin{array}{l}\text { NCT01098487 } \\
\text { [11] }\end{array}$ & ITP & $\begin{array}{l}\mathrm{N}=167 \\
\text { Adults } \\
\text { (Females:104+ } \\
\text { Males:58) }\end{array}$ & $\begin{array}{l}\text { Phase } 4 \\
\text { Open } \\
\text { label }\end{array}$ & $\begin{array}{l}\text { Eltrombopag } \\
\text { olamine orally }\end{array}$ & $\begin{array}{l}\text { CITPwas found to be } \\
\text { a pro-thrombotic } \\
\text { disease. }\end{array}$ & $\begin{array}{l}\text { (42 S.A.E +114 A.E /162) } \\
\text { Menorrhagia, lethargy, headache, } \\
\text { abdominal pain, fatigue, chest pain, } \\
\text { vomiting, anaemia, diarrhoea. }\end{array}$ \\
\hline 4. & $\begin{array}{l}\text { 2008-2015 } \\
\text { (3 months) }\end{array}$ & $\begin{array}{l}\text { NCT00699140 } \\
{[12]}\end{array}$ & ITP & $\begin{array}{l}\mathrm{N}=18 \\
\text { Adults } \\
\text { (Females:12 } \\
+ \\
\text { Males:6) }\end{array}$ & $\begin{array}{l}\text { Phase } 3 \\
\text { Open } \\
\text { label }\end{array}$ & $\begin{array}{l}\text { IGIV3I Grifols } \\
\text { intravenoulsy }\end{array}$ & $\begin{array}{l}\text { Safe and effective; } \\
\text { Well tolerated }\end{array}$ & $\begin{array}{l}\text { (2 S.A.E +12 A.E /18) } \\
\text { Leukopenia, Thrombosis, } \\
\text { Haemoglobin decreased, } \\
\text { Hypertension, Ecchymosis, Petechiae, } \\
\text { Headache, Pyrexia, nausea, vomiting, } \\
\text { abdominal pain. }\end{array}$ \\
\hline
\end{tabular}


South American Journal of Clinical Research

Volume 3, Issue 1, 2016

\begin{tabular}{|c|c|c|c|c|c|c|c|c|}
\hline 5. & $\begin{array}{l}\text { 2012-2014 } \\
\text { (37 weeks) }\end{array}$ & $\begin{array}{l}\text { NCT01520909 } \\
{[13]}\end{array}$ & ITP & $\begin{array}{l}\mathrm{N}=92 \\
\text { Children } \\
\text { (Females:44 } \\
+ \\
\text { Males:48) }\end{array}$ & $\begin{array}{l}\text { Phase } 3 \\
\text { Double } \\
\text { blinded }\end{array}$ & $\begin{array}{l}\text { Eltrombopag } \\
\text { orally }\end{array}$ & $\begin{array}{l}\text { Production of a } \\
\text { sustained platelet } \\
\text { response in } 40 \% \text { of } \\
\text { patients with ITP }\end{array}$ & $\begin{array}{l}\text { (18 S.A.E +68 A.E /87) } \\
\text { Epistaxis, abdominal pain, diarrhoea, } \\
\text { rash, headache, back pain, rhinitis, } \\
\text { nasopharyngitis, pyrexia, nausea and } \\
\text { vomiting. }\end{array}$ \\
\hline 6. & $\begin{array}{l}\text { 2009-2014 } \\
\text { (3 years) }\end{array}$ & $\begin{array}{l}\text { NCT00907478 } \\
{[14]}\end{array}$ & ITP & $\begin{array}{l}\mathrm{N}=169 \\
\text { Adults } \\
\text { (Females:114+ } \\
\text { Males:55) }\end{array}$ & $\begin{array}{l}\text { Phase } 4 \\
\text { Open } \\
\text { label }\end{array}$ & $\begin{array}{l}\text { Romiplostim } \\
\text { subcutaneous } \\
\text { injection }\end{array}$ & $\begin{array}{l}\text { Low incidence of } \\
\text { both collagen and } \\
\text { increased reticulin }\end{array}$ & $\begin{array}{l}\text { (56 S.A.E +154 A.E /169) } \\
\text { Pulmonary embolism, Epistaxis, } \\
\text { urinary tract infection, } \\
\text { Thrombocytopenia, Hypertension, } \\
\text { Haematoma, Petechiae, Ecchymosis, } \\
\text { Epistaxis, cough, headache, dizziness, } \\
\text { Arthralgia, Contusion, fatigue, } \\
\text { nausea, vomiting, diarrhoea, } \\
\text { Thrombocytopenia. }\end{array}$ \\
\hline 7. & $\begin{array}{l}\text { 2009-2014 } \\
\text { (31 weeks) }\end{array}$ & $\begin{array}{l}\text { NCT00908037 } \\
\text { [15] }\end{array}$ & ITP & $\begin{array}{l}\mathrm{N}=82 \\
\text { Children } \\
\text { (Females:48 } \\
+ \\
\text { Males:34) }\end{array}$ & $\begin{array}{l}\text { Phase } 2 \\
\text { Double } \\
\text { blinded }\end{array}$ & $\begin{array}{l}\text { Eltrombopag } \\
\text { orally }\end{array}$ & $\begin{array}{l}\text { Increased platelet } \\
\text { counts and reduced } \\
\text { clinically significant } \\
\text { bleeding in children } \\
\text { with persistent or } \\
\text { CITP }\end{array}$ & $\begin{array}{l}\text { Utricaria, Eczema, Petechiae, Rash, } \\
\text { nasal congestion, Oropharyngeal } \\
\text { pain, cough, Epistaxis, Insomnia, } \\
\text { nughtmare, headache, fatigue. }\end{array}$ \\
\hline 8. & $\begin{array}{l}\text { 2007-2014 } \\
\text { (52 weeks) }\end{array}$ & $\begin{array}{l}\text { NCT00475423 } \\
{[16]}\end{array}$ & ITP & $\begin{array}{l}\mathrm{N}=122 \\
\text { Adults } \\
\text { (Females:70 } \\
+ \\
\text { Males:52) }\end{array}$ & $\begin{array}{l}\text { Phase } 2 \\
\text { Open } \\
\text { label }\end{array}$ & $\begin{array}{l}\text { Rituximab } \\
\text { [MabThera/Rituxa } \\
\text { n] Intravenously }\end{array}$ & $\begin{array}{l}\text { Well tolerated with } \\
\text { no safety concerns. } \\
\text { Efficacy not } \\
\text { observed. }\end{array}$ & $\begin{array}{l}\text { (12 S.A.E +62 A.E /122) } \\
\text { Stress, platelet count decreased, back } \\
\text { pain, Diarhhoea, Thrombocytopenia, } \\
\text { Hypertension, rash, Petechiae, } \\
\text { Epistaxis, headache, contusion, } \\
\text { fatigue, vomiting. }\end{array}$ \\
\hline 9. & $\begin{array}{l}2007-2014 \\
\text { (24 months) }\end{array}$ & $\begin{array}{l}\text { NCT00706342 } \\
\text { [17] }\end{array}$ & ITP & $\begin{array}{l}\mathrm{N}=18 \\
\text { Adults } \\
\text { (Females:10 }\end{array}$ & $\begin{array}{l}\text { Phase } 2 \\
\text { Open } \\
\text { label }\end{array}$ & $\begin{array}{l}\text { Fostamatinib } \\
\text { Disodium / } \\
\text { R935788 orally }\end{array}$ & $\begin{array}{l}\text { Increased and } \\
\text { maintained the } \\
\text { platelet count in ITP }\end{array}$ & $\begin{array}{l}\text { (8 S.A.E +18 A.E /18) } \\
\text { Diarrhea, nausea, vomiting, } \\
\text { dehdration, ear pain, blurred vision, }\end{array}$ \\
\hline
\end{tabular}


South American Journal of Clinical Research Volume 3, Issue 1, 2016

\begin{tabular}{|c|c|c|c|c|c|c|c|c|}
\hline & & & & $\begin{array}{l}+ \\
\text { Males:8) }\end{array}$ & & & patients & $\begin{array}{l}\text { dry eye, nausea, vomiting, fatigue, } \\
\text { abdominal pain, chest pain, } \\
\text { contusion, hypertension, headache, } \\
\text { diziness, anxiety, depression, } \\
\text { Epistaxis, Ecchymosis, Petechiae. }\end{array}$ \\
\hline 10. & $\begin{array}{l}\text { 2006-2014 } \\
\text { (63 days) }\end{array}$ & $\begin{array}{l}\text { NCT00426270 } \\
{[18]}\end{array}$ & ITP & $\begin{array}{l}\mathrm{N}=116 \\
\text { Adults } \\
\text { (Females:74 } \\
+ \\
\text { Males:42) }\end{array}$ & $\begin{array}{l}\text { Phase } 3 \\
\text { Open } \\
\text { label }\end{array}$ & $\begin{array}{l}\text { Octagam } 10 \% \\
\text { intravenously }\end{array}$ & $\begin{array}{l}\text { Well tolerated and } \\
\text { effective in patients } \\
\text { with ITP }\end{array}$ & $\begin{array}{l}\text { (14 S.A.E +79 A.E /116) } \\
\text { Headache, Thrombocytopenia, } \\
\text { Idiopathic thrombocytopenic purpura, } \\
\text { nausea, Pyrexia, Headache, } \\
\text { Hypertension. }\end{array}$ \\
\hline 11. & $\begin{array}{l}\text { 2005-2014 } \\
\text { (24 weeks) }\end{array}$ & $\begin{array}{l}\text { NCT00770562 } \\
{[19]}\end{array}$ & ITP & $\begin{array}{l}\mathrm{N}=103 \\
\text { Adults } \\
\text { (Females:60 } \\
+ \\
\text { Males:41) }\end{array}$ & $\begin{array}{l}\text { Phase } 3 \\
\text { Open } \\
\text { label }\end{array}$ & $\begin{array}{l}\text { Rituximab Orally } \\
\text { and Intravenously }\end{array}$ & $\begin{array}{l}\text { Safe and effective. } \\
\text { Combination therapy } \\
\text { improved platelet } \\
\text { counts. }\end{array}$ & $\begin{array}{l}\text { (4 S.A.E + } 62 \text { A.E /101) } \\
\text { Rib fracture, Anemia, Abdominal } \\
\text { pain, constipation, gingival bleeding, } \\
\text { insomnia, diziness, headache, cough, } \\
\text { Petechiae, hypertension. }\end{array}$ \\
\hline 12. & $\begin{array}{l}\text { 2007-2012 } \\
\text { (90 days) }\end{array}$ & $\begin{array}{l}\text { NCT00504075 } \\
\text { [20]. }\end{array}$ & CITP & $\begin{array}{l}\mathrm{N}=35 \\
18-70 \text { yrs. } \\
\text { Adults (Females: } \\
26 \\
+ \\
\text { Males:9) }\end{array}$ & $\begin{array}{l}\text { Phase } 3 \\
\text { Open } \\
\text { label }\end{array}$ & $\begin{array}{l}\text { Gammaplex, } \\
\text { intravenously } \\
\text { immunoglobulin }\end{array}$ & $\begin{array}{l}\text { Effective treatment } \\
\text { and well tolerated } \\
\text { Achieved a very high } \\
\text { concentration of } \\
\text { serum }\end{array}$ & $\begin{array}{l}\text { (4 S.A.E + } 21 \text { A.E /35) } \\
\text { Headache, } \\
\text { Vomiting, Pyrexia, Palpitations, } \\
\text { Nausea, Arthralgia, Diarrhoea, } \\
\text { Dehydration, anemia, abdominal } \\
\text { pain, Gingival bleeding, Petechiae, } \\
\text { hypertension. }\end{array}$ \\
\hline 13. & $\begin{array}{l}\text { 2005-2012 } \\
\text { (201 weeks) }\end{array}$ & $\begin{array}{l}\text { NCT00508820 } \\
\text { [21] }\end{array}$ & ITP & $\begin{array}{l}\mathrm{N}=407 \\
\text { Adults } \\
\text { (Females:244+ } \\
\text { Males:163) }\end{array}$ & $\begin{array}{l}\text { Phase } 3 \\
\text { Open } \\
\text { label }\end{array}$ & $\begin{array}{l}\text { Romiplostim } \\
\text { subcutaneous } \\
\text { injection }\end{array}$ & $\begin{array}{l}\text { Safe, effictive and } \\
\text { well tolerated. }\end{array}$ & $\begin{array}{l}\text { (122 S.A.E +343 A.E/407 ) } \\
\text { Haemorrhage, Petechiae, Epistaxis, } \\
\text { Thrombocytopenia, Anaemia, } \\
\text { Abdominal pain, Diarrhoea, Gingival } \\
\text { bleeding, nausea, vomiting, cheat }\end{array}$ \\
\hline
\end{tabular}


South American Journal of Clinical Research

Volume 3, Issue 1, 2016

\begin{tabular}{|c|c|c|c|c|c|c|c|c|}
\hline & & & & & & & & $\begin{array}{l}\text { pain, fatigue, decreased appetite, } \\
\text { diziness, headache, anxiety, } \\
\text { depression, Hypertension. }\end{array}$ \\
\hline 14. & $\begin{array}{l}\text { 2009-2011 } \\
\text { (42 days) }\end{array}$ & $\begin{array}{l}\text { NCT01133860 } \\
{[22]}\end{array}$ & $\begin{array}{l}\text { Blood } \\
\text { Platelet } \\
\text { Disorders }\end{array}$ & $\begin{array}{l}\mathrm{N}=12 \\
\text { Children } \\
+ \\
\text { Adults } \\
\text { (Females:7 } \\
+ \\
\text { Males:5) }\end{array}$ & $\begin{array}{l}\text { Phase } 2 \\
\text { Open } \\
\text { label }\end{array}$ & $\begin{array}{l}\text { Eltrombopag } \\
\text { orally }\end{array}$ & $\begin{array}{l}\text { Bleeding tendency } \\
\text { disappeared }\end{array}$ & $\begin{array}{l}\text { (2 S.A.E /12) } \\
\text { Headache, dry mouth. }\end{array}$ \\
\hline 15. & $\begin{array}{l}2008-2011 \\
\text { (981 days) }\end{array}$ & $\begin{array}{l}\text { NCT00828750 } \\
\text { [23] }\end{array}$ & ITP & $\begin{array}{l}\mathrm{N}=19 \\
\text { Adults (Females: } \\
12 \\
+ \\
\text { Males:7) }\end{array}$ & $\begin{array}{l}\text { Phase } 3 \\
\text { Open } \\
\text { label }\end{array}$ & $\begin{array}{l}\text { Eltrombopag } \\
\text { orally }\end{array}$ & $\begin{array}{l}\text { Safe, well tolerated, } \\
\text { and effective in long } \\
\text { term use in chronic } \\
\text { ITP. }\end{array}$ & $\begin{array}{l}\text { (6 S.A.E +19 A.E /19) } \\
\text { Cataract, abdominal pain, } \\
\text { Menorrhagia, Lumbar spinal stenosis, } \\
\text { anaemia, blurred vision, Diarrhoea, } \\
\text { chest pain, fatigue, Pyrexia, } \\
\text { Nasopharyngitis, headache, insomnia, } \\
\text { hypertension. }\end{array}$ \\
\hline 16. & $\begin{array}{l}2004-2011 \\
\text { (285 weeks) }\end{array}$ & $\begin{array}{l}\text { NCT00116688 } \\
\text { [24] }\end{array}$ & ITP & $\begin{array}{l}\mathrm{N}=313 \\
\text { Children (20) } \\
+ \\
\text { Adults (291) } \\
\text { (Females:190+ } \\
\text { Males:123) }\end{array}$ & $\begin{array}{l}\text { Phase } 3 \\
\text { Open } \\
\text { label }\end{array}$ & $\begin{array}{l}\text { Romiplostim } \\
\text { subcutaneous } \\
\text { injection }\end{array}$ & $\begin{array}{l}\text { Safe and well- } \\
\text { tolerated }\end{array}$ & $\begin{array}{l}\text { (119 S.A.E + } 288 \text { A.E / 313) } \\
\text { Influenza (child), anaemia, abdominal } \\
\text { pain, diarrhoea, nausea, vomiting, } \\
\text { toothache, fatigue, anxity, depression, } \\
\text { hypertension. }\end{array}$ \\
\hline 17. & $\begin{array}{l}2004-2011 \\
\text { (29 days) }\end{array}$ & $\begin{array}{l}\text { NCT00168038 } \\
\text { [25] }\end{array}$ & ITP & $\begin{array}{l}\mathrm{N}=58 \\
\text { Children } \\
+ \\
\text { Adults } \\
\text { (Female:34 }\end{array}$ & $\begin{array}{l}\text { Phase } 3 \\
\text { Open } \\
\text { label }\end{array}$ & $\begin{array}{l}\text { Immunoglobulin } \\
\text { Intravenously }\end{array}$ & $\begin{array}{l}\text { Effective in } \\
\text { increasing platelet } \\
\text { count, reduced } \\
\text { bleeding events and } \\
\text { was well tolerated. }\end{array}$ & $\begin{array}{l}\text { (3 S.A.E + } 52 \text { A.E /57) } \\
\text { Sudden hearing loss, Meningitis } \\
\text { aseptic, dizziness, headache, } \\
\text { Petechiae, Epistaxis, contusion, } \\
\text { pyrexia, nausea, vomiting. }\end{array}$ \\
\hline
\end{tabular}


South American Journal of Clinical Research Volume 3, Issue 1, 2016

\begin{tabular}{|c|c|c|c|c|c|c|c|c|}
\hline & & & & $\begin{array}{l}+ \\
\text { Male: } 23)\end{array}$ & & & & \\
\hline 18. & $\begin{array}{l}2007-2010 \\
\text { (12 weeks) }\end{array}$ & $\begin{array}{l}\text { NCT00603642 } \\
{[26]}\end{array}$ & ITP & $\begin{array}{l}\mathrm{N}=34 \\
\text { Adults (Females: } \\
24 \\
+ \\
\text { Males: } 10)\end{array}$ & $\begin{array}{l}\text { Phase } 3 \\
\text { Doublebl } \\
\text { inded }\end{array}$ & $\begin{array}{l}\text { Romiplostim } \\
\text { subcutaneous } \\
\text { injection }\end{array}$ & $\begin{array}{l}\text { Safe and well } \\
\text { tolerated. }\end{array}$ & $\begin{array}{l}\text { (3 S.A.E +16 A.E/34) } \\
\text { Thrombocytopenia, Gastrointestinal } \\
\text { haemorrhage, Petechiae, headache, } \\
\text { Nasopharyngitis, headache. }\end{array}$ \\
\hline 19. & $\begin{array}{l}2007-2010 \\
\text { (12 weeks) }\end{array}$ & $\begin{array}{l}\text { NCT00515203 } \\
{[27]}\end{array}$ & ITP & $\begin{array}{l}\mathrm{N}=22 \\
\text { Children } \\
\text { (Females: } 6 \\
+ \\
\text { Males: } 16 \text { ) }\end{array}$ & $\begin{array}{l}\text { Phase1|P } \\
\text { hase } 2 \\
\text { Double } \\
\text { blinded }\end{array}$ & $\begin{array}{l}\text { Romiplostim } \\
\text { subcutaneous } \\
\text { injection }\end{array}$ & $\begin{array}{l}\text { Increased platelet } \\
\text { counts in 88\% of } \\
\text { children with ITP } \\
\text { and was well- } \\
\text { tolerated and } \\
\text { apparently safe. }\end{array}$ & $\begin{array}{l}\text { (1 S.A.E +21 A.E /22) } \\
\text { Influenza, sepsis, pyrexia, abdominal } \\
\text { pain, vomiting, contusion, headache, } \\
\text { Petechiae. }\end{array}$ \\
\hline 20. & $\begin{array}{l}\text { 2007-2009 } \\
\text { (6 weeks) }\end{array}$ & $\begin{array}{l}\text { NCT00424177 } \\
\text { [28] }\end{array}$ & ITP & $\begin{array}{l}\mathrm{N}=66 \\
\text { Adults (Females: } \\
45 \\
+ \\
\text { Males: } 21)\end{array}$ & $\begin{array}{l}\text { Phase } 2 \\
\text { Open } \\
\text { label }\end{array}$ & $\begin{array}{l}\text { Eltrombopag } \\
\text { orally }\end{array}$ & $\begin{array}{l}\text { Significantly reduced } \\
\text { bleeding in adult } \\
\text { patients with chronic } \\
\text { ITP. }\end{array}$ & $\begin{array}{l}\text { (7 S.A.E + } 55 \text { A.E/66) } \\
\text { Epistaxis, } \\
\text { anxiety, insomnia, headache, fatigue, } \\
\text { vomiting, diarrhoea }\end{array}$ \\
\hline 21. & $\begin{array}{l}\text { 2007-2009 } \\
\text { (26 weeks) }\end{array}$ & $\begin{array}{l}\text { NCT00540423 } \\
\text { [29]. }\end{array}$ & CITP & $\begin{array}{l}\mathrm{N}=23 \\
\text { Greater than } 20 \\
\text { years } \\
\text { Adults } \\
\text { (Females:15 } \\
+ \\
\text { Males: 8) }\end{array}$ & $\begin{array}{l}\text { Phase } 3 \\
\text { Randomi } \\
\text { zed } \\
\text { double } \\
\text { blinded } \\
\text { study }\end{array}$ & $\begin{array}{l}\text { Eltrombopag } \\
\text { orally }\end{array}$ & $\begin{array}{l}\text { Well tolerated. } \\
\text { Decreased bleeding. }\end{array}$ & $\begin{array}{l}\text { (7 S.A.E +19 A.E /23) } \\
\text { Hemorrhagic diathesis, headache, } \\
\text { fatigue, back pain, nausea. }\end{array}$ \\
\hline 22. & 2006-2009 & NCT00415532 & ITP & $\mathrm{N}=234$ & Phase 3 & Romiplostim & Greater HRQOL & (64 S.A.E +196 A.E /234) \\
\hline
\end{tabular}


South American Journal of Clinical Research

Volume 3, Issue 1, 2016

\begin{tabular}{|c|c|c|c|c|c|c|c|c|}
\hline & (52 weeks) & [30] & & $\begin{array}{l}\text { Adults (Females: } \\
131 \\
+ \\
\text { Males:103) }\end{array}$ & $\begin{array}{l}\text { Open } \\
\text { label }\end{array}$ & $\begin{array}{l}\text { subcutaneous } \\
\text { injection }\end{array}$ & $\begin{array}{l}\text { improvements } \\
\text { observed than those } \\
\text { receiving Standard of } \\
\text { care. }\end{array}$ & $\begin{array}{l}\text { Thrombocytopenia, abdominal pain, } \\
\text { pneumonia, contusion, anemia, } \\
\text { vertigo, constipation, diarrhoea, } \\
\text { nausea, vomiting, fatigue, Pyrexia, } \\
\text { Petechiae, hypertension. }\end{array}$ \\
\hline 23. & $\begin{array}{l}2006-2009 \\
\text { (6 months) }\end{array}$ & $\begin{array}{l}\text { NCT00370331 } \\
{[31]}\end{array}$ & ITP & $\begin{array}{l}\mathrm{N}=197 \\
\text { Adults (Females: } \\
136 \\
+ \\
\text { Males:61) }\end{array}$ & $\begin{array}{l}\text { Phase } 3 \\
\text { Doublebl } \\
\text { inded }\end{array}$ & $\begin{array}{l}\text { Eltrombopag } \\
\text { orally }\end{array}$ & $\begin{array}{l}\text { Well tolerated; } \\
\text { Improved bleeding } \\
\text { scores with improved } \\
\text { physical and mental } \\
\text { health }\end{array}$ & $\begin{array}{l}\text { (27 S.A.E + } 176 \text { A.E /196) } \\
\text { Catarct, Conjunctival haemorrhage, } \\
\text { Diarrhoea, Nausea, vomiting, } \\
\text { constipation, fatigue, Headache, back } \\
\text { pain, Epistaxis, insomnia. }\end{array}$ \\
\hline 24. & $\begin{array}{l}\text { 2006-2009 } \\
\text { (36 months) }\end{array}$ & $\begin{array}{l}\text { NCT00454857 } \\
{[32]}\end{array}$ & ITP & $\begin{array}{l}\mathrm{N}=326 \\
\text { Adults } \\
\text { (Females:195+ } \\
\text { Males: 131) } \\
\text { (older than } 18 \\
\text { yrs.) }\end{array}$ & $\begin{array}{l}\text { observati } \\
\text { onal }\end{array}$ & $\begin{array}{l}\text { Patient-reported } \\
\text { Outcome } \\
\text { Questionnaires: } \\
\text { survey }\end{array}$ & $\begin{array}{l}\text { Acute and chronic } \\
\text { idiopathic } \\
\text { thrombocytopenic } \\
\text { purpura seemed to be } \\
\text { distinct illnesses } \\
\text { defined by age, } \\
\text { platelet count, } \\
\text { bleeding symptoms, } \\
\text { and the presence of } \\
\text { acute illness before } \\
\text { diagnosis. }\end{array}$ & $\begin{array}{l}\text { Deaths }=5 ; \\
\text { None serious side-effects. }\end{array}$ \\
\hline 25. & $\begin{array}{l}2003-2009 \\
\text { ( }<6 \text { weeks) }\end{array}$ & $\begin{array}{l}\text { NCT00220727 } \\
{[33]}\end{array}$ & ITP & $\begin{array}{l}\mathrm{N}=8 \\
\text { Children } \\
+ \\
\text { Adults } \\
\text { (Females:7 } \\
+ \\
\text { Males:1) } \\
(12-75)\end{array}$ & $\begin{array}{l}\text { Phase } 2 \\
\text { Open } \\
\text { label }\end{array}$ & $\begin{array}{l}\text { Immune Globulin } \\
\text { Intravenously. } \\
\text { [Human], } 10 \% \\
\text { Caprylate/Chroma } \\
\text { tography Purified }\end{array}$ & $\begin{array}{l}\text { No signs of } \\
\text { hemolysis; } \\
\text { Safe and Well } \\
\text { tolerated. }\end{array}$ & $\begin{array}{l}\text { (8 A.E /8) } \\
\text { Arthralgia, dizziness, rash, Headache. }\end{array}$ \\
\hline
\end{tabular}


S.A.E: Severe Adverse Events

A.E: Adverse Events

\section{Results}

Table 1. Data Extraction and Results Retrieval Interpretation [9-33].

\begin{tabular}{|c|c|c|}
\hline S.No. & Characteristics & Trials and Subjects \\
\hline 1. & Total number ITP patients & 2773 no. of patients \\
\hline 2. & \begin{tabular}{lc}
\multicolumn{2}{l}{ Diagnosis year by calendar period: } \\
a. & $2003-2009$ \\
b. & $2007-2010$ \\
c. & $2004-2011$ \\
d. & $2005-2012$ \\
e. & $2005-2014$ \\
f. & $2008-2015$ \\
g. & $2010-2016$ \\
\end{tabular} & $\begin{array}{l}6 \text { trials } \\
2 \text { trials } \\
4 \text { trials } \\
2 \text { trials } \\
7 \text { trials } \\
3 \text { trials } \\
1 \text { trials }\end{array}$ \\
\hline 3. & \begin{tabular}{ll}
\multicolumn{2}{l}{ Age at diagnosis: } \\
a. & Children \\
b. & Adults \\
c. & Children + Adults
\end{tabular} & $\begin{array}{l}196 \text { no. of patients } \\
2186 \text { no. of patients } \\
389 \text { no. of patients }\end{array}$ \\
\hline 4. & $\begin{array}{ll}\text { Sex: } & \\
\text { a. } & \text { Male } \\
\text { b. } & \text { Female } \\
\end{array}$ & $\begin{array}{l}\text { 1082no. of patients } \\
\text { 1689no. of patients }\end{array}$ \\
\hline 5. & Drug induced thrombocytopenia & 8 trials \\
\hline 6. & Total no. of deaths occured & 43 no. of patients \\
\hline 7. & Phase 1 & 22 no. of patients \\
\hline 8. & Phase 2 & 405 no. of patients \\
\hline 9. & Phase 3 & 1649 no. of patients \\
\hline 10. & Phase 4 & 393 no. of patients \\
\hline 11. & $\begin{array}{l}\text { Total no. of randomized controlled trials } \\
\text { from } 2003 \text { to } 2016\end{array}$ & $11 / 25$ trials \\
\hline 12. & $\begin{array}{l}\text { Total no. of non-randomized controlled } \\
\text { trials from } 2003 \text { to } 2016\end{array}$ & $13 / 25$ trials \\
\hline 13. & $\begin{array}{l}\text { Total no. of observational studies from } \\
2003 \text { to } 2016\end{array}$ & $1 / 25$ trials \\
\hline 14. & Experimental drugs studied & 9 treatments \\
\hline 15. & $\begin{array}{l}\text { Safety and efficacy profile achieved in } \\
\text { how many clinical trials }\end{array}$ & 20 trials \\
\hline 16. & Trials showed increase in platelet count & 10 trials \\
\hline 17. & $\begin{array}{l}\text { Total no. of Serious Adverse events } \\
\text { associated with the interventional } \\
\text { treatment }\end{array}$ & 527 no. of patients \\
\hline 18. & $\begin{array}{l}\text { Total no. of Adverse events associated } \\
\text { with the interventional treatment }\end{array}$ & 1854 no. of patients \\
\hline
\end{tabular}

\section{Discussion}

The literature review revealed that side effects from corticosteroids intervention were found to be bothersome. In studies performed from the year 2009-2016, it was observed that when Romiplostim subcutaneous injection was administered as an interventional drug in Children and Adults, there was an increase in the platelet counts in early stage ITP patients by marked low incidence of collagen and increased reticulin [9, 14, 21, 24, 26, 27, 30]. Moreover, the drug was safe and well tolerated in Phase 1, Phase2, Phase3 and Phase 4 clinical trials. Apart from this, Quality of life of ITP patients improved significantly [9, 14, 
21, 24, 26, 27, 30]. Romiplostim known to be a thrombopoietin peptide mimetic agent had demonstrated an increase in the platelet count in the majority of patients with ITP, both in splenectomized and non-splenectomized patients [34, 35, 36, 37]. In up to 5 years of follow up, its safety profile was favorable, that suggestedRomiplostim to be a novel therapeutic agent in patients with ITP with the potentiation of induced tolerance and reduced use of steroidsthereby achieved the reduced rate of treatment failure and the need for splenectomy which was accompanied by greater improvements in quality of life [34, 35, 36, 37]. Platelet responses were achieved by $>90 \%$ of the patients, typically within 1-2 weeks of the initiation of romiplostim treatment [35]. Romiplostim was found to be well tolerated among children and adults who suffered from ITP [38].

On the other hand, a pilot study with R788(Fostamatinib), an orally administered Syk kinase inhibitor that was a prodrug of R406, was initiated to explore the safety and efficacy of treatment for patients with chronic ITP [17, 39]. R788 was found to be effective in maintaining adequate platelet counts and in reducing the need for rescue medications in most patients[17, 39]. In the future, it might be an alternative to treatment with newer thrombopoiesis-stimulating agents or be combined with them to take advantage of the different mechanisms of therapeutic effect $[17,39]$.

In a phase 4 study of administration of IgPRo10 (Privigen) intravenous interventional drug, it was observed that a set of antibodies frequently bound to the Red Blood cells of ITP patients $[10,40]$. In that two-day regimen study of Privigen on ITP patients, it was found to be effective in increased platelet count, reduced bleeding events and was well tolerated [10, 40].

In studies performed from the year 2009-2015, it was observed that when Eltrombopag oral tablets were administered as an interventional drug in Children and Adults, there was a marked production of a sustained platelet response in platelet counts with reduced bleeding in ITP patients thereby suggesting ITP as a Pro-thrombotic disease [11, 13, 15, 22, 23, 28, 29, 31]. Moreover, the drug was safe and well tolerated in Phase2, Phase3 and Phase4 clinical trials[11, 13, 15, 22, 23, 28, 29, 31]. Apart from this, marked improvement in Quality of life of ITP patients was observed [11, 13, 15, 22, 23, 28, 29, 31]. Eltrombopag was observed to be effective for management of chronic immune thrombocytopenia, by giving once daily and could be particularly beneficial for patients who did not respond to splenectomy or previous treatment [41].

In a 2015 Phase 3 study performed, it was observed that the intravenous administration of IGIV3I to ITP adult patients, produced significant results within 3 to 6 days of its infusion [12]. The drug was safe and well tolerated [12].

In another interventional drug, Rituximab (2014), it was observed that the addition of rituximab to a single course of dexamethasone therapy improved outcomes in ITP patients when used as first-line or salvage therapy [16, 19, 37, 42].

Another interventional drug, Octagam $10 \%$ was found to be efficacious in both chronic ITP and newly diagnosed patients, as measured by platelet count and resolution of bleeding and had a good tolerability profile with no unexpected safety issues [18, 43].

In a study performed on intravenous administration of Immunoglobulin on ITP patients, it was observed that the interventional drug achieved increase in platelet count along with reduced bleeding events with no sign of hemolysis [20, 25, 33]. The drug was found to be effective and well tolerated among ITP patients [20, 25, 33]. Besides, Bone marrow aspiration was highly recommended for ITP patients who were found to be resistant to Intravenous Ig with persistant thrombocytopenia [3].

In a nutshell, from the above data retrieved it could be concluded that there were only limited number of clinical trials in context of ITP management as seen from the year 20032016. Moreover, trials conducted on children were comparatively low as comparedto that of adults. It was evident from the data retrieved that females were observed to be more sensitive and at high risk for developing ITP along with the increase and advancement in age. The mortality rate during the trials were observed to be $1.5 \%$ which was unknown if it occured 
due to the treatment or due to the progression of disease itself. The present review encompassed the study of administration of 9 interventional drugs either in the oral form or in the I.V. form. On the whole, it could be concluded that the administration of Romiplostim and Eltrombopag proved to be safe and effective for long term use in ITP patients with minimal side effects. All the interventional drugs studied produced significant and promising results, however, were found to be associated with certain adverse events such as, Nausea, vomiting, fatigue, insomia, nightmares, pyrexia, abdominal pain, Diarrhoea, Headache, Petechiae, vertigo, hypertension, contusion, diziness, Epistaxis, anemia, anxiety and depression which ultimately had a greater impact on the quality of life of ITP patients [9-33].

This study was influential in the general opinion that $30 \times 109 / \mathrm{L}$ was a reasonable cut-off for treating ITP. An international consensus report stated that treatment was rarely indicated in patients with platelet counts above $50 \times 109 / \mathrm{L}$ in the absence of bleeding due to platelet dysfunction or other hemostatic defect, trauma, or surgery. Although this number was not supported by evidence-based data [1].

\section{Conclusion}

The management of patients with ITP should consider the age of the patient, the severity of the illness, and the anticipated natural history. ITP was observed as a serious autoimmune disease with little epidemiological evidence on its burden, risk factors, and comorbidities. The present review provided a robust population-based estimate of the incidence and management of ITP in the adults and children that confirmed the incidence of ITP increased with age and was found to be higher among women than men. At present, most treatment protocols concentrated on the reduction of platelet destruction, and the drugs used were usually immunosuppressives. However, other drugs might be used in the near future if the TPO mimetic proved safe and effective in the various trials currently in progress. The literature synthesis and themes from the focused group data suggested that decreased platelet counts, disease symptoms, and treatment side effects influenced multiple domains of HRQOL for ITP patients, that includedfatigue, muscle cramps, and psychiatric problems such as depressionthereby, impactingemotional and functional health, work life, social and leisure activities, and reproductive health. Thus, by evaluating the therapeutic strategies for ITP, it was observed that ITP affected various areas of HRQOL. In conclusion, this study revealed that most chronic ITP-patients had a benign clinical course. Low platelet count and frequency of wet and dry purpura did not predict a higher risk of hospital admissions, but age did. The current century had brought a number of informative prospective clinical trials in ITP. Clinical studies as well as basic cellular and molecular investigations had also advanced our understanding of the pathogenesis of ITP. To date, the treatment of these disorders in adults had been disappointing, in part because of a lack of understanding of the underlying pathophysiology in this diverse group of disorders.

\section{Future directions}

According to the current practice guidelines, it had been stated that women with ITP did not require testing for maternal platelet antibodies; however, Percutaneous umbilical vein blood sampling (PUBS) or fetal scalp vein sampling should be done to measure the fetal platelet count in order to, predict the risk of neonatal bleeding. Therefore, there is a major need for adequate counseling and care for pregnant ITP-patients. Moreover, there were no tests that aided in diagnosing ITP with certainty. A more rational, consistent and effective approach was required for a clear understanding of the natural history of the disease and the development of targeted treatments in carefully designed randomized studies. Areas that required focus in the future included the development of sensitive and specific laboratory tests for diagnosis validated bleeding scores and health related quality of life assessments for patients affected with ITP. 
South American Journal of Clinical Research

Volume 3, Issue 1, 2016

\section{Limitations of the study}

a. Quality of the data collected and retrieved through searching the database was highly variable and conflicting.

b. The cost-effectiveness of the treatment regimen intervention and the travel cost tools were not included in the study.

c. Recommendations regarding thresholds for safe platelet counts for specific medical interventions might vary widely.

d. Reported deaths could not necessarily be attributed to ITP related or treatment related.

\section{References}

[1.] Arnold, D.M., Dentali, F., Crowther, M.A., Meyer, R.M., Cook, R.J., Sigouin, C., et al. (2007). Systematic Review: Efficacy and Safety of Rituximab for Adults with Idiopathic Thrombocytopenic Purpura. Ann Intern Med., 146:25-33. DOI: http://10.7326/0003-4819-146-1-200701020-00006

[2.] Bussel, J.B., Buchanan, G.R., Nugent, D.J., Gnarra, D.J., Bomgaars, L.R., Blanchette, V.S., Wang, Y.M., Nie, K., Jun, S. (2011). A randomized, double-blind study of romiplostim to determine its safety and efficacy in children with immune thrombocytopenia. Blood, 118(1). DOI: http://10.1182/blood2010-10-313908.

[3.] Cheng, G., Saleh, M.N., Marcher, C., Vasey, S., Mayer, B., Aivado, M., Arning, M., Stone, N.L., Bussel, J.B. (2010). Eltrombopag for management of chronic immune thrombocytopenia (RAISE): a 6month, randomised, phase 3 study. The Lancet, 377(9763); 393-402. DOI: http://dx.doi.org/10.1016/S0140-6736(10)60959-2

[4.] ClinicalTrials.gov [Internet]. Amgen. 2016 January 27-Identifier NCT01143038, Interventional Study in Adults With Immune Thrombocytopenia Purpura (ITP) Receiving Romiplostim; Accessed on: 2016 June 14; Accessed from:

https://clinicaltrials.gov/ct2/show/NCT01143038?term=Idiopathic+Thrombocytopenic+Purpura\&recr= Closed\&no_unk=Y\&rslt=With\&type=Intr\&rank=14

[5.] ClinicalTrials.gov [Internet]. CSL Behring. 2016 March 10-Identifier NCT01390649, A Safety Study of Intravenous Immunoglobulin in Patients With Chronic Immune Thrombocytopenic Purpura (ITP); Accessed on: 2016 June 14; Accessed from:

https://clinicaltrials.gov/ct2/show/NCT01390649?term=Idiopathic+Thrombocytopenic+Purpura\&recr= Closed\&no_unk=Y\&rslt=With\&type=Intr\&rank=15

[6.] ClinicalTrials.gov [Internet]. GlaxoSmithKline. 2015 February 26-Identifier NCT01098487, A Longitudinal 2-year Bone Marrow Study of Eltrombopag in Previously Treated Adults, With Chronic Immune (Idiopathic) Thrombocytopenic Purpura (ITP); Accessed on: 2016 June 14; Accessed from: https://clinicaltrials.gov/ct2/show/NCT01098487?term=Idiopathic+Thrombocytopenic+Purpura\&recr= Completed\&no_unk=Y\&rslt=With\&type=Intr\&rank=33

[7.] ClinicalTrials.gov [Internet]. Instituto Grifols, S.A. 2016 January 9-Identifier NCT00699140, Clinical Trial in Patients Diagnosed With Immune Thrombocytopenic Purpura (ITP); Accessed on: 2016 June 14; Accessed from:

https://clinicaltrials.gov/ct2/show/NCT00699140?term=Idiopathic+Thrombocytopenic+Purpura\&recr= Completed\&no_unk=Y\&rslt=With\&type $=$ Intr\&rank=20

[8.] ClinicalTrials.gov [Internet]. GlaxoSmithKline. 2015 February 19-Identifier NCT01520909, Study of a New Medication for Childhood Chronic Immune Thrombocytopenia (ITP), a Blood Disorder of Low Platelet Counts That Can Lead to Bruising Easily, Bleeding Gums, and/or Bleeding Inside the Body. (PETIT2); Accessed on: 2016 June 14; Accessed from:

https:/clinicaltrials.gov/ct2/show/NCT01520909?term=Idiopathic+Thrombocytopenic+Purpura\&recr= Closed\&no_unk=Y\&rslt=With\&type=Intr\&rank=8

[9.] ClinicalTrials.gov [Internet]. Amgen. 2014 December 19-Identifier NCT00907478, Study on Bone Marrow Morphology in Adults Receiving Romiplostim for Treatment of Thrombocytopenia Associated With Immune Thrombocytopenia Purpura (ITP); Accessed on: 2016 June 14; Accessed from: 
https://clinicaltrials.gov/ct2/show/NCT00907478?term=Idiopathic+Thrombocytopenic+Purpura\&recr= Closed\&no_unk $=$ Y\&rslt=With\&type $=$ Intr\&rank $=10$

[10.] ClinicalTrials.gov [Internet]. GlaxoSmithKline. 2015 March 19-Identifier NCT00908037, Efficacy and Safety Study of Eltrombopag in Pediatric Patients With Thrombocytopenia From Chronic Idiopathic Thrombocytopenic Purpura (ITP) (PETIT); Accessed on: 2016 June 14; Accessed from: https://clinicaltrials.gov/ct2/show/NCT00908037?term=Idiopathic+Thrombocytopenic+Purpura\&recr= Closed\&no_unk=Y\&rslt=With\&type=Intr\&rank=13

[11.] ClinicalTrials.gov [Internet]. Hoffmann-La Roche. 2015 February 4-Identifier NCT00475423, A Study of MabThera (Rituximab) in Patients With Idiopathic Thrombocytopenic Purpura; Accessed on: 2016 June 14; Accessed from:

https://clinicaltrials.gov/ct2/show/study/NCT00475423?term=Idiopathic+Thrombocytopenic+Purpura \&recr=Closed\&no_unk=Y\&rslt=With\&type=Intr\&rank=4\&view=results

[12.] ClinicalTrials.gov [Internet]. Rigel Pharmaceuticals. 2016 May 3-Identifier NCT00706342, Pilot Study of Fostamatinib Disodium/R935788 for the Treatment of Adult Refractory Immune Thrombocytopenic Purpura (ITP); Accessed on: 2016 June 14; Accessed from:

https://clinicaltrials.gov/ct2/show/NCT00706342?term=Idiopathic+Thrombocytopenic+Purpura\&recr= Closed\&no_unk=Y\&rslt=With\&type=Intr\&rank=16

[13.] ClinicalTrials.gov [Internet]. Octapharma. 2014 July 28-Identifier NCT00426270, Clinical Study to Evaluate the Efficacy and Safety of Octagam 10\% in Idiopathic Thrombocytopenic Purpura in Adults; Accessed on: 2016 June 14; Accessed from:

https://clinicaltrials.gov/ct2/show/NCT00426270?term=Idiopathic+Thrombocytopenic+Purpura\&recr= Closed\&no_unk=Y\&rslt=With\&type=Intr\&rank=5

[14.] ClinicalTrials.gov [Internet]. Hoffmann-La Roche. 2014 October 21-Identifier NCT00770562, A Study Evaluating the Addition of MabThera (Rituximab) to Standard Treatment in Patients With Idiopathic Thrombocytopenic Purpura (ITP); Accessed on: 2016 June 14; Accessed from:

https://clinicaltrials.gov/ct2/show/NCT00770562?term=Idiopathic+Thrombocytopenic+Purpura\&recr= Closed\&no_unk=Y\&rslt=With\&type=Intr\&rank=7

[15.] ClinicalTrials.gov [Internet]. Bio Products Laboratory. 2013 February 26-Identifier NCT00504075, A Study to Find How Safe and Effective GAMMAPLEX ${ }^{\circledR}$ is in Subjects With Chronic Idiopathic Thrombocytopenic Purpura (ITP); Accessed on: 2016 June 14; Accessed from:

https://clinicaltrials.gov/ct2/show/NCT00504075?term=NCT00504075\&rank=1

[16.] ClinicalTrials.gov [Internet]. Amgen. 2016 February 22-Identifier NCT00508820, An Open Label Study of Romiplostim in Adult Thrombocytopenic Subjects With ITP; Accessed on: 2016 June 14; Accessed from:

https://clinicaltrials.gov/ct2/show/NCT00508820?term=Idiopathic+Thrombocytopenic+Purpura\&recr= Closed\&no_unk=Y\&rslt=With\&type=Intr\&rank=17

[17.] ClinicalTrials.gov [Internet]. IRCCS Policlinico S. Matteo. 2011 July 22-Identifier NCT01133860, Efficacy of Eltrombopag to Improve Thrombocytopenia of MYH9-related Disease; Accessed on: 2016 June 14; Accessed from:

https://clinicaltrials.gov/ct2/show/NCT01133860?term=Idiopathic+Thrombocytopenic+Purpura\&recr= Completed\&no_unk=Y\&rslt=With\&type=Intr\&rank=23

[18.] ClinicalTrials.gov [Internet]. GlaxoSmithKline. 2011 October 31-Identifier NCT00828750, Clinical Evaluation of Eltrombopag in Chronic Idiopathic Thrombocytopenic Purpura (ITP); Accessed on: 2016 June 14; Accessed from:

https://clinicaltrials.gov/ct2/show/NCT00828750?term=Idiopathic+Thrombocytopenic+Purpura\&recr= Closed\&no_unk=Y\&rslt=With\&type=Intr\&rank=3

[19.] ClinicalTrials.gov [Internet]. Amgen. 2013 November 25-Identifier NCT00116688, Open Label Extension Study of Romiplostim (AMG 531) in Thrombocytopenic Patients With Immune (Idiopathic) Thrombocytopenic Purpura (ITP); Accessed on: 2016 June 14; Accessed from:

https://clinicaltrials.gov/ct2/show/NCT00116688?term=Idiopathic+Thrombocytopenic+Purpura\&recr= Completed\&no_unk=Y\&rslt=With\&type=Intr\&rank=21 
South American Journal of Clinical Research

Volume 3, Issue 1, 2016

[20.] ClinicalTrials.gov [Internet]. CSL Behring. 2011 October 18-Identifier NCT00168038, Treatment of Chronic Immune Thrombocytopenic Purpura (ITP) With Intravenous Immunoglobulin IgPro10; Accessed on: 2016 June 14; Accessed from:

https:/clinicaltrials.gov/ct2/show/NCT00168038?term=Idiopathic+Thrombocytopenic+Purpura\&recr= Closed\&no_unk=Y\&rslt=With\&type=Intr\&rank=6

[21.] ClinicalTrials.gov [Internet]. Amgen. 2011 February 4-Identifier NCT00603642, P3 Study to Evaluate Efficacy and Safety of AMG 531 in Thrombocytopenic Japanese Subjects With Immune (Idiopathic) Thrombocytopenic Purpura; Accessed on: 2016 June 14; Accessed from:

https://clinicaltrials.gov/ct2/show/NCT00603642?term=Idiopathic+Thrombocytopenic+Purpura\&recr= Closed\&no_unk=Y\&rslt=With\&type=Intr\&rank=18

[22.] ClinicalTrials.gov [Internet]. Amgen. 2014 July 18-Identifier NCT00515203, Safety and Efficacy Study of Romiplostim (AMG 531) to Treat ITP in Pediatric Subjects; Accessed on: 2016 June 14; Accessed from:

https://clinicaltrials.gov/ct2/show/NCT00515203?term=Idiopathic+Thrombocytopenic+Purpura\&recr= Completed\&rslt=With\&rank=19

[23.] ClinicalTrials.gov [Internet]. GlaxoSmithKline. 2013 July 25-Identifier NCT00424177, Repeated Exposure to Eltrombopag in Adults With Idiopathic Thrombocytopenic Purpura (REPEAT); Accessed on: 2016 June 14; Accessed from:

https://clinicaltrials.gov/ct2/show/NCT00424177?term=Idiopathic+Thrombocytopenic+Purpura\&recr= Closed\&no_unk=Y\&rslt=With\&type=Intr\&rank=9

[24.] ClinicalTrials.gov [Internet]. Amgen. 2014 July 18-Identifier NCT00415532, Romiplostim (AMG 531) Versus Medical Standard of Care for Immune (Idiopathic) Thrombocytopenic Purpura; Accessed on: 2016 June 14; Accessed from:

https://clinicaltrials.gov/ct2/show/NCT00415532?term=Idiopathic+Thrombocytopenic+Purpura\&recr= Closed\&no_unk=Y\&rslt=With\&type=Intr\&rank=12

[25.] ClinicalTrials.gov [Internet]. GlaxoSmithKline. 2012 November 21-Identifier NCT00370331, RAISE: Randomized Placebo-Controlled Idiopathic Thrombocytopenic Purpura (ITP) Study With Eltrombopag (RAISE); Accessed on: 2016 June 14; Accessed from:

https://clinicaltrials.gov/ct2/show/NCT00370331?term=Idiopathic+Thrombocytopenic+Purpura\&recr= Completed\&rslt $=$ With\&rank=20

[26.] ClinicalTrials.gov [Internet]. Amgen. 2013 October 23-Identifier NCT00454857, Retrospective \& Prospective Observational Study of Patients With Immune (Idiopathic) Thrombocytopenic Purpura (ITP); Accessed on: 2016 June 14; Accessed from:

https://clinicaltrials.gov/ct2/show/NCT00454857?term=Idiopathic+Thrombocytopenic+Purpura\&recr= Completed\&rslt=With\&type $=$ Obsr\&rank $=1$

[27.] ClinicalTrials.gov [Internet]. Grifols Therapeutics Inc. 2016 March 28-Identifier NCT00220727, Rapid Infusion Of Immune Globulin Intravenous (IGIV) In Patients With ITP; Accessed on: 2016 June 14; Accessed from:

https://clinicaltrials.gov/ct2/show/NCT00220727?term=Idiopathic+Thrombocytopenic+Purpura\&recr= Completed\&no_unk=Y\&rslt=With\&type=Intr\&rank=97

[28.] Cooper, N., Terrinoni, I., \& Newland, A. (2012). The efficacy and safety of romiplostim in adult patients with chronic immune thrombocytopenia. Therapeutic Advances in Hematology, 3(5), 291-298. DOI: http://doi.org/10.1177/2040620712453596

[29.] Immune Thrombocytopenic Purpura (ITP): A New Look at an Old Disorder. (2010). Indiana Hemophilia \& Thrombosis Center. Accessed from:

http://www.ihtc.org/wp-content/uploads/2010/05/Final\%20BT\%20Spring\%202010\%20PRINT.pdf

[30.] Janssens, A., Tarantino, M., Bird, R.J., Mazzucconi, M.G., Boccia, R.V., Fernández, M.F., Kozak, T., Steurer, M., Boekhorst, P., Dillingham, K., Kreuzbauer, G., Woodard, P. (2015). Romiplostim Treatment in Adults with Immune Thrombocytopenia of Varying Duration and Severity. Acta Haematol, 134(4):215-28. Accessed from: http://www.ncbi.nlm.nih.gov/pubmed/26066765

[31.] Johnsen, J. (2012). Pathogenesis in immune thrombocytopenia: new insights. Hematology, 306312. DOI: http://10.1182/asheducation-2012.1.306 
[32.] Mathias, S.D., Gao, S. K., Miller, K. L., Cella, D., Snyder, C., Turner, R., Wu, A., Bussel, J.B., George, J.N., McMillan, R., Wysocki, D.K., and Nichol, J. L. (2008). Impact of chronic Immune Thrombocytopenic Purpura (ITP) on health-related quality of life: a conceptual model starting with the patient perspective. BioMed Central, 6:13. DOI: http://10.1186/1477-7525-6-13

[33.] Matzdorff, A. C., Arnold, G., Salama, A., Ostermann, H., Eberle, S., \& Hummler, S. (2011). Advances in ITP - Therapy and Quality of Life - A Patient Survey. PLoS ONE, 6(11), e27350. DOI: http://doi.org/10.1371/journal.pone.0027350

[34.] McCRAE, K. (2011). Immune thrombocytopenia: No longer "idiopathic.". Cleveland Clinic Journal of Medicine, 78(6), 358-373. DOI: http://doi.org/10.3949/ccjm.78gr.10005

[35.] Newland, A., Godeau, B., Priego, V., Viallard, J.F., López Fernández, M.F., Orejudos, A., Eisen, M. (2016). Remission and platelet responses with romiplostim in primary immune thrombocytopenia: final results from a phase 2 study. Br J Haematol, 172(2):262-73. DOI: http://10.1111/bjh.13827.

[36.] Podolanczuk, A., Alan, H., Lazarus, Andrew, R., Crow, Grossbard, E., Bussel, J.B. (2009). Of mice and men: an open-label pilot study for treatment of immune thrombocytopenic purpura by an inhibitor of Syk. Blood, 113, 14. DOI: http://10.1182/blood-2008-07-166439

[37.] Provan, D., \& Newland, A. (2002). Fifty years of idiopathic thrombocytopenic purpura (ITP): management of refractory itp in adults. British Journal of Haematology, 118(4), 933-944. DOI: http://10.1046/j.1365-2141.2002.03669.x

[38.] Robak, T., Mainau, C., Pyringer, B., Chojnowski, K., Warzocha, K., Dmoszynska, A., Straub, J., Imbach, P. (2010). Efficacy and safety of a new intravenous immunoglobulin $10 \%$ formulation in patients with immune thrombocytopenia. Hematology, 15(5). DOI: http://10.1179/102453310x:271901099:867

[39.] Robak, T., Salama, A., Kovaleva, L., Vyhovska, Y., Davies, S.V., Mazzucconi, M.G., Zenker, O., Kiessling, P. (2009). International Privigen in ITP Study Group. Efficacy and safety of Privigen, a novel liquid intravenous immunoglobulin formulation, in adolescent and adult patients with chronic immune thrombocytopenic purpura. Hematology, 14(4):227-36. DOI: http://10.1179/102453309X439773.

[40.] Stasi, R. (2011). Pathophysiology and therapeutic options in primary immune thrombocytopenia. Blood Transfusion, 9(3), 262-273. DOI: http://doi.org/10.2450/2010.0080-10

[41.] Tomiyama, Y., Miyakawa, Y., Okamoto, S., Katsutani, S., Kimure, A., Okoshi, Y., Ninomiya, H., Kosugi, H., Nomura, S., Ozaki, K., Ikeda, Y., Hattori, T., Katsura, K., Kanakur, Y. (2012). A lower starting dose of eltrombopag is efficacious in Japanese patients with previously treated chronic immune thrombocytopenia. Journal of Thrombosis and Haemostasis, 10: 799-806. DOI: http://10.1111/j.15387836.2012.04695.X

[42.] Tomiyama, Y., Miyakawa, Y., Okamoto, S., Katsutani, S., Kimure, A., Okoshi, Y., Ninomiya, H., Kosugi, H., Nomura, S., Ozaki, K., Ikeda, Y., Hattori, T., Katsura, K., Kanakur, Y. (2012). A lower starting dose of eltrombopag is efficacious in Japanese patients with previously treated chronic immune thrombocytopenia. Journal of Thrombosis and Haemostasis, 10: 799-806. DOI: http://10.1111/j.15387836.2012.04695.x

[43.] Warrier, R., \& Chauhan, A. (2012). Management of Immune Thrombocytopenic Purpura: An Update. The Ochsner Journal, 12(3), 221-227. Accessed from: http://www.ncbi.nlm.nih.gov/pmc/articles/PMC3448244/.

[44.] Zaja, F., Baccarani, M., Mazza, P., Bocchia, M., Gugliotta, L., Zaccaria, A., Vianelli, N., Defina, M., Tieghi, A., Amadori, S., Campagna, S., Ferrara, F. Angelucci, E., Usala, E., Cantoni, S., Visani, G., Fornaro, A., Rizzi, R., Stefano, V.D., Casulli, F., Battista, M.L., Isola, M., Soldano, F., Gamba, E., and Fanin, R. (2010). Dexamethasone plus rituximab yields higher sustained response rates than dexamethasone monotherapy in adults with primary immune thrombocytopenia. Blood, 115(14); DOI: http://10.1182/blood-2009-07-229815 